\section{Metabolic alkalosis in hospitalised COVID-19 patients: A window to the pathogenesis?}

To the Editor: South Africa has documented $>600000$ confirmed COVID-19 cases within the 6 months since notification of the first case.$^{[1]}$ During this influx of COVID-19 patients, we have observed an under-reported finding on the arterial blood gas of some hospitalised patients, namely metabolic alkalosis with or without hypoxaemia. No known causes of alkalosis were present in these patients, and as it was clinically associated with SARS-CoV-2 infection, the finding soon became known as the 'COVID gas' among frontline clinicians.

We conducted a prospective cohort study to determine the prevalence and significance of otherwise unexplained metabolic alkalosis in 255 consecutive patients admitted with suspected COVID-19. Standard bicarbonate $\left(\mathrm{sHCO}_{3}\right)$ was used as a marker for the metabolic component. ${ }^{[2]}$

One hundred and fifty patients tested SARS-CoV-2-positive. The median $\mathrm{sHCO}_{3}$ was 26.7 (interquartile range (IQR) 23.95 - 29.2) $\mathrm{mmol} / \mathrm{L}, 27.6$ (IQR 25.2 - 29.4) $\mathrm{mmol} / \mathrm{L}$ and 25.2 (IQR 22.1 - 28.1) $\mathrm{mmol} / \mathrm{L}$ for the whole cohort, the SARS-CoV-2-positive group and the SARS-CoV-2-negative group, respectively. The median $\mathrm{pH}$ was 7.46 (IQR 7.4 - 7.49), 7.47 (IQR 7.45 - 7.5) and 7.42 (IQR 7.34 - 7.47) for the respective groups. Metabolic alkalosis $\left(\mathrm{sHCO}_{3} \geq 28 \mathrm{mmol} / \mathrm{L}\right.$ and $\mathrm{pH} 27.45$ ) was significantly more common in SARS-CoV-2positive than SARS-CoV-2-negative patients (60/150 (40\%) v. 17/105 (16\%); $p<0.001$ ). The median potassium level was 4.0 (IQR 4.0 - 4.5) $\mathrm{mmol} / \mathrm{L}$ and 5.0 (IQR $4.0-5.0$ ) $\mathrm{mmol} / \mathrm{L}$ in the SARS-CoV-2-positive and negative groups, respectively. Thirteen patients in the SARSCoV-2-positive group (9\%) had hypokalaemia $(<3.5 \mathrm{mmol} / \mathrm{L})$ and 8 had both hypokalaemia and metabolic alkalosis. Urine samples obtained from 4 of these patients all showed an increased potassiumto-creatinine ratio, with a median of $3.1 \mathrm{mmol} / \mathrm{mmol}$. The 'COVID gas' had moderate specificity (84\%) but poor sensitivity (40\%) for COVID-19 diagnosis. In the 143 COVID-19 patients with available outcomes, neither the presence nor the absence of metabolic alkalosis predicted survival.

We present this unusual observation to assess opinion regarding the consistency of this finding in other centres, and to gather opinion as to the potential causes and clinical significance of the pronounced metabolic alkalosis. We speculate that the metabolic alkalosis may be due to excess mineralocorticoid-like effects or hypokalaemia, either from renal or gastrointestinal losses. ${ }^{[3]}$ Chen et al. ${ }^{[4]}$ reported a 53\% prevalence of hypokalaemia in COVID-19 patients. The renin-angiotensin system (RAS) effects are antagonised by actions of angiotensin-converting enzyme 2 (ACE2). SARS-CoV-2 binds and degrades ACE2, thereby potentially reducing its counter-regulatory effects. ${ }^{[5]}$ Increased RAS activity with increased angiotensin II and aldosterone effects may therefore promote distal nephron sodium reabsorption and enhanced urinary potassium excretion. Our cohort predominantly had normal concentrations of serum potassium, probably because the acute nature of SARS-CoV-2 infection does not affect total body potassium stores significantly.

The unusual presence of metabolic alkalosis on admission in a significant number of patients admitted with COVID-19 is an unreported finding requiring multicentre investigation to unravel the pathophysiology of the disease, including the significance of the acid-base abnormalities we observed.

\section{Jacques Rood}

Department of Medicine, Tygerberg Hospital and Faculty of Medicine and Health Sciences, Stellenbosch University, Cape Town, South Africa

\section{Razeen Davids}

Division of Nephrology, Department of Medicine, Tygerberg Hospital and Faculty of Medicine and Health Sciences, Stellenbosch University, Cape Town, South Africa

\section{Alwyn le Roux}

Department of Medical Imaging and Clinical Oncology, Tygerberg Hospital and Faculty of Medicine and Health Sciences, Stellenbosch University, Cape Town, South Africa

\section{Melissa du Plessis}

Division of General Medicine, Department of Medicine, Tygerberg Hospital and Faculty of Medicine and Health Sciences, Stellenbosch University, Cape Town, South Africa

\section{Arifa Parker}

Divisions of Infectious Diseases and General Medicine, Department of Medicine, Tygerberg Hospital and Faculty of Medicine and Health Sciences, Stellenbosch University, Cape Town, South Africa

\section{Brian W Allwood}

Division of Pulmonology, Department of Medicine, Tygerberg Hospital and Faculty of Medicine and Health Sciences, Stellenbosch University, Cape Town, South Africa

\section{Hans W Prozesky}

Division of Infectious Diseases, Department of Medicine, Tygerberg Hospital and Faculty of Medicine and Health Sciences, Stellenbosch University, Cape Town, South Africa

\section{Coenraad F N Koegelenberg}

Division of Pulmonology, Department of Medicine, Tygerberg Hospital and Faculty of Medicine and Health Sciences, Stellenbosch University, Cape Town, South Africa

\section{Jantjie J Taljaard}

Division of Infectious Diseases, Department of Medicine, Tygerberg Hospital and Faculty of Medicine and Health Sciences, Stellenbosch University, Cape Town, South Africa

jjt@sun.ac.za

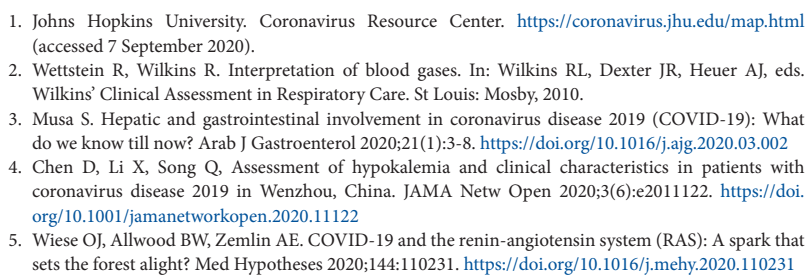
(accessed 7 September 2020).

Wettstein R, Wilkins R. Interpretation of blood gases. In: Wilkins RL, Dexter JR, Heuer AJ, eds. Wilkins' Clinical Assessment in Respiratory Care. St Louis: Mosby, 2010.

3. Musa S. Hepatic and gastrointestinal involvement in coronavirus disease 2019 (COVID-19): What do we know till now? Arab J Gastroenterol 2020;21(1):3-8. https://doi.org/10.1016/..ajg.2020.03.002

4. Chen D, Li X, Song Q, Assessment of hypokalemia and clinical characteristics in patients with coronavirus disease 2019 in Wenzhou, China. JAMA Netw Open 2020;3(6):e2011122. https://doi. org/10.1001/jamanetworkopen.2020.11122

5. Wiese OJ, Allwood BW, Zemlin AE. COVID-19 and the renin-angiotensin system (RAS): A spark that sets the forest alight? Med Hypotheses 2020;144:110231. https://doi.org/10.1016/j.mehy.2020.110231

S Afr Med J 2020;110(11):1060. https://doi.org/10.7196/SAMJ.2020.v110i11.15287 